\title{
More Identities for Fibonacci and Lucas Octonions
}

\author{
Nurettin Irmak* and Abdullah Açıkel
}

\begin{abstract}
Keywords: Fibonacci Octonions; Lucas Octonions, octonions

AMS Subject Classification (2020): 11D09; 11B39; 11R52

${ }^{*}$ Corresponding author
\end{abstract}

In this paper, we give a new approach to obtain identities for Fibonacci and Lucas octonions.

\section{Introduction}

$O$ is an eight-dimensional non-associative and non-commutative division algebra over $\mathbb{R}$. A natural basis of this algebra as a space over $\mathbb{R}$ is formed by the elements

$$
e_{0}=1, e_{1}=i, e_{2}=j, e_{3}=k, e_{4}=e, e_{5}=i e, e_{6}=j e, e_{7}=k e
$$

where $i^{2}=j^{2}=k^{2}=-1, i j=k=-j i, j k=i=-k j, k i=j=-i k$ and $i j k=-1$.

The multiplication table for the basis of $O$ is

\begin{tabular}{|l||c|c|c|c|c|c|c|c|}
\hline$\cdot$ & 1 & $e_{1}$ & $e_{2}$ & $e_{3}$ & $e_{4}$ & $e_{5}$ & $e_{6}$ & $e_{7}$ \\
\hline \hline 1 & 1 & $e_{1}$ & $e_{2}$ & $e_{3}$ & $e_{4}$ & $e_{5}$ & $e_{6}$ & $e_{7}$ \\
\hline$e_{1}$ & $e_{1}$ & -1 & $e_{3}$ & $-e_{2}$ & $e_{5}$ & $-e_{4}$ & $-e_{7}$ & $e_{6}$ \\
\hline$e_{2}$ & $e_{2}$ & $-e_{3}$ & -1 & $e_{1}$ & $e_{6}$ & $e_{7}$ & $-e_{4}$ & $-e_{5}$ \\
\hline$e_{3}$ & $e_{3}$ & $e_{2}$ & $-e_{1}$ & -1 & $e_{7}$ & $-e_{6}$ & $e_{5}$ & $-e_{4}$ \\
\hline$e_{4}$ & $e_{4}$ & $-e_{5}$ & $e_{6}$ & $-e_{7}$ & -1 & $e_{1}$ & $e_{2}$ & $e_{3}$ \\
\hline$e_{5}$ & $e_{5}$ & $e_{4}$ & $-e_{7}$ & $e_{6}$ & $-e_{1}$ & -1 & $-e_{3}$ & $e_{2}$ \\
\hline$e_{6}$ & $e_{6}$ & $e_{7}$ & $e_{4}$ & $-e_{5}$ & $-e_{2}$ & $e_{3}$ & -1 & $-e_{1}$ \\
\hline$e_{7}$ & $e_{7}$ & $-e_{6}$ & $e_{5}$ & $e_{4}$ & $-e_{3}$ & $-e_{2}$ & $e_{1}$ & -1 \\
\hline
\end{tabular}

Together with this notation, a octonion $p$ takes the form

$$
p=\sum_{i=0}^{7} p_{i} e_{i}
$$

where $p_{i} \in \mathbb{R}$. Every octonion $p$ can be written as the sum of two parts, real and imaginary parts.

$$
p=\operatorname{Re}(p)+\operatorname{Im}(p) .
$$

This definition gives that $\operatorname{Re}(p)=p_{0}$ and $\operatorname{Im}(p)=\sum_{i=1}^{7} p_{i} e_{i}$. The conjugate of $p, \bar{p}$, is defined

$$
\bar{p}=\operatorname{Re}(p)-\operatorname{Im}(p) .
$$

For $p, q \in O$, the identities

$$
\overline{\bar{p}}=p, \overline{(p+q)}=\bar{p}+\bar{q}, \overline{p q}=\overline{q p}
$$

Received : 12-07-2019, Accepted : 01-05-2020 
follow.

For $n \geq 2$, the Fibonacci and Lucas sequences are defined as

$$
F_{n}=F_{n-1}+F_{n-2}, \quad F_{0}=0, F_{1}=1
$$

and

$$
L_{n}=L_{n-1}+L_{n-2}, \quad L_{0}=2, L_{1}=1,
$$

respectively. There are lots of amazing identities belongs to Fibonacci and Lucas numbers. For the details, we refer the book of T. Koshy ([3]).

Keçilioğlu and Akkuş [2] gave the definitions of the Fibonacci and Lucas octonions as

$$
Q_{n}=\sum_{j=0}^{7} F_{n+j} e_{j}, H_{n}=\sum_{j=0}^{7} L_{n+j} e_{j},
$$

respectively. Moreover, they present several identities about the Fibonacci and Lucas octonions. The binet formulas of these octonions are used in their paper to get identities.

Up to now, the identities for both Fibonacci quaternion Lucas quaternions and Fibonacci octonion Lucas octonions are obtained by the using binet formulas or special identities for Fibonacci and Lucas numbers. Irmak [1] gives an alternative approach to obtain identities. He used the matrix methods for Fibonacci and Lucas quaternions.

In this paper, we give several identities for Fibonacci and Lucas octonions by using Irmak's approach.

Before going further, we need a square matrix $W$ as

$$
W=\left(\begin{array}{cc}
Q_{1} & Q_{0} \\
Q_{0} & Q_{-1}
\end{array}\right)
$$

Since the algebra $O$ is non-commutative, we need two different determinant definition. Let $A$ be a octonion square matrix. Denote $A$ by,

$$
A=\left(\begin{array}{ll}
a_{11} & a_{12} \\
a_{21} & a_{22}
\end{array}\right)
$$

where $a_{i j} \in O$ for $i=1,2$ and $j=1,2$. The determinant of $A$, $\operatorname{det} A$, is defined by

$$
\begin{aligned}
\operatorname{det} A & =\operatorname{det}\left(\begin{array}{cc}
a_{11} & a_{12} \\
a_{21} & a_{22}
\end{array}\right) \\
& =a_{11} a_{22}-a_{12} a_{21} .
\end{aligned}
$$

The above definition is called rule "multiplication from above to down below". Since the set of all quaternion is not commutative, another product direction can be defined. Namely, The definition

$$
\begin{aligned}
\operatorname{Det} A & =\operatorname{det}\left(\begin{array}{cc}
a_{11} & a_{12} \\
a_{21} & a_{22}
\end{array}\right) \\
& =a_{22} a_{11}-a_{21} a_{12}
\end{aligned}
$$

is called the rule "multiplication from down below to above" (For details, see the book [4], section 9.11.)

The Fibonacci matrix $U$ is known that

$$
U=\left(\begin{array}{ll}
1 & 1 \\
1 & 0
\end{array}\right)
$$

which yields that

$$
U^{n}=\left(\begin{array}{cc}
F_{n+1} & F_{n} \\
F_{n} & F_{n-1}
\end{array}\right) .
$$

If we multiply the matrices $W$ and $U^{n}$, we get Fibonacci octonion matrix (entries are Fibonacci octonions). Namely, we obtain the following fact

$$
W U^{n}=U^{n} W=\left(\begin{array}{cc}
Q_{n+1} & Q_{n} \\
Q_{n} & Q_{n-1}
\end{array}\right) .
$$




\section{Main Result}

First theorem is about the Cassini identity belongs to Fibonacci and Lucas octonions. Since there are two different determinant definitions of a octonion matrix, we give the different Cassini identities.

Theorem 2.1. (First type Cassini identity) For $n \geq 1$, we have the identities

$$
Q_{n-1} Q_{n+1}-Q_{n}^{2}=(-1)^{n}\left(2 Q_{1}-\left(14 e_{5}+12 e_{6}+7 e_{7}\right)\right)
$$

and

$$
H_{n-1} H_{n+1}-H_{n}^{2}=5(-1)^{n-1}\left(2 Q_{1}-\left(14 e_{5}+12 e_{6}+7 e_{7}\right)\right) .
$$

Proof. By (1.3)

$$
\begin{aligned}
U^{n} W & =\left(\begin{array}{cc}
F_{n+1} & F_{n} \\
F_{n} & F_{n-1}
\end{array}\right)\left(\begin{array}{cc}
Q_{1} & Q_{0} \\
Q_{0} & Q_{-1}
\end{array}\right) \\
& =\left(\begin{array}{cc}
Q_{n+1} & Q_{n} \\
Q_{n} & Q_{n-1}
\end{array}\right)
\end{aligned}
$$

follows. After taking determinant (1.2) together with the identity $F_{n+1} F_{n-1}-F_{n}^{2}=(-1)^{n}$, we obtain

$$
\begin{aligned}
Q_{n-1} Q_{n+1}-Q_{n}^{2} & =\left(F_{n+1} F_{n-1}-F_{n}^{2}\right)\left(Q_{-1} Q_{1}-Q_{0}^{2}\right) \\
& =(-1)^{n}\left(2 Q_{1}-\left(14 e_{5}+12 e_{6}+7 e_{7}\right)\right) .
\end{aligned}
$$

as claimed. Since $F_{n-1}+F_{n+1}=L_{n}$, then we write

$$
\begin{aligned}
\left(U^{n-1}+U^{n+1}\right) W & =\left(\begin{array}{cc}
L_{n+1} & L_{n} \\
L_{n} & L_{n-1}
\end{array}\right)\left(\begin{array}{cc}
Q_{1} & Q_{0} \\
Q_{0} & Q_{-1}
\end{array}\right) \\
& =\left(\begin{array}{cc}
H_{n+1} & H_{n} \\
H_{n} & H_{n-1}
\end{array}\right) .
\end{aligned}
$$

Applying the equation (1.2) gives that

$$
\begin{aligned}
H_{n-1} H_{n+1}-H_{n}^{2} & =\left(L_{n+1} L_{n-1}-L_{n}^{2}\right)\left(Q_{-1} Q_{1}-Q_{0}^{2}\right) \\
& =5(-1)^{n-1}\left(2 Q_{1}-\left(14 e_{5}+12 e_{6}+7 e_{7}\right)\right) .
\end{aligned}
$$

Now, we use the first definition of determinant (1.1) to obtain the second type Cassini identity.

Theorem 2.2. (Second type Cassini identity) For $n \geq 1$, we have the identities

$$
Q_{n+1} Q_{n-1}-Q_{n}^{2}=(-1)^{n}\left(2 Q_{-1}+14 e_{5}+18 e_{6}+7 e_{7}\right)
$$

and

$$
H_{n+1} H_{n-1}-H_{n}^{2}=5(-1)^{n-1}\left(2 Q_{-1}+14 e_{5}+18 e_{6}+7 e_{7}\right) .
$$

Proof. Since the equation (2.1) holds, then the definition 1.1 yields that

$$
\begin{aligned}
Q_{n+1} Q_{n-1}-Q_{n}^{2} & =\left(F_{n-1} F_{n+1}-F_{n}^{2}\right)\left(Q_{1} Q_{-1}-Q_{0}^{2}\right) \\
& =(-1)^{n}\left(2 Q_{-1}+14 e_{5}+18 e_{6}+7 e_{7}\right) .
\end{aligned}
$$

Similarly, we get

$$
H_{n+1} H_{n-1}-H_{n}^{2}=5(-1)^{n-1}\left(2 Q_{-1}+14 e_{5}+18 e_{6}+7 e_{7}\right) .
$$

Theorem 2.3. For $n, m \geq 1$ integers, then

$$
Q_{m+n}=F_{m+1} Q_{n}+F_{m} Q_{n-1}
$$

follows. 
Proof. The fact $U^{m+n} F=U^{m}\left(U^{n} F\right)$ yields that

$$
\begin{aligned}
U^{m+n} W & =\left(\begin{array}{cc}
Q_{m+n+1} & Q_{m+n} \\
Q_{m+n} & Q_{m+n-1}
\end{array}\right) \\
& =U^{m}\left(U^{n} W\right) \\
& =\left(\begin{array}{cc}
F_{m+1} & F_{m} \\
F_{m} & F_{m-1}
\end{array}\right)\left(\begin{array}{cc}
Q_{n+1} & Q_{n} \\
Q_{n} & Q_{n-1}
\end{array}\right) .
\end{aligned}
$$

Equating the first row second column entries of two matrix in (2.2) yields that

$$
Q_{m+n}=F_{m+1} Q_{n}+F_{m} Q_{n-1}
$$

Since $F_{m-2}+F_{m-1}=F_{m}$ and $Q_{m+n+1}+Q_{m+n-1}=H_{m+n}$ holds, we get the following identity as a corollary.

Corollary 2.1. For positive integers $m$ and $n$, we get

$$
H_{m+n}=L_{m+1} Q_{n}+L_{m} Q_{n-1}
$$

Theorem 2.4. For $n, m \geq 1$ integers, we have

$$
Q_{m+1} Q_{n}+Q_{m} Q_{n-1}=\sum_{i=0}^{7} Q_{m+n+i} e_{i} .
$$

Proof. The identity $F\left(T^{m+n} F\right)=\left(F T^{m}\right)\left(T^{n} F\right)$ yields that

$$
\begin{aligned}
& \left(\begin{array}{cc}
Q_{1} & Q_{0} \\
Q_{0} & Q_{-1}
\end{array}\right)\left(\begin{array}{cc}
Q_{m+n+1} & Q_{m+n} \\
Q_{m+n} & Q_{m+n-1}
\end{array}\right) \\
= & \left(\begin{array}{cc}
Q_{m+1} & Q_{m} \\
Q_{m} & Q_{m-1}
\end{array}\right)\left(\begin{array}{cc}
Q_{n+1} & Q_{n} \\
Q_{n} & Q_{n-1}
\end{array}\right)
\end{aligned}
$$

If we equalize the first row and second column elements in the equation (2.4), we obtain the claimed result.

We deduce the following matrix equation system by the equation (2.3).

$$
\left(\begin{array}{cc}
H_{m+n+1} & H_{m+n} \\
H_{m+n} & H_{m+n-1}
\end{array}\right)=\left(\begin{array}{cc}
L_{m+1} & L_{m} \\
L_{m} & L_{m-1}
\end{array}\right)\left(\begin{array}{cc}
Q_{n+1} & Q_{n} \\
Q_{n} & Q_{n-1}
\end{array}\right)
$$

If we multiply the equation (2.5) with the matrix $W$ from left side, we deduce

$$
\begin{aligned}
& \left(\begin{array}{ll}
Q_{1} & Q_{0} \\
Q_{0} & Q_{-1}
\end{array}\right)\left(\begin{array}{cc}
H_{m+n+1} & H_{m+n} \\
H_{m+n} & H_{m+n-1}
\end{array}\right) \\
= & \left(\left(\begin{array}{cc}
Q_{1} & Q_{0} \\
Q_{0} & Q_{-1}
\end{array}\right)\left(\begin{array}{cc}
L_{m+1} & L_{m} \\
L_{m} & L_{m-1}
\end{array}\right)\right)\left(\begin{array}{cc}
Q_{n+1} & Q_{n} \\
Q_{n} & Q_{n-1}
\end{array}\right) \\
= & \left(\begin{array}{cc}
H_{m+1} & H_{m} \\
H_{m} & H_{m-1}
\end{array}\right)\left(\begin{array}{cc}
Q_{n+1} & Q_{n} \\
Q_{n} & Q_{n-1}
\end{array}\right) .
\end{aligned}
$$

Equating the first row and second column element, we obtain the following theorem.

Theorem 2.5. For $m, n \geq 1$, we get

$$
H_{m+1} Q_{n}+H_{m} Q_{n-1}=\sum_{i=0}^{7} H_{m+n+1+i} e_{i} .
$$


Let define the conjugate matrix of $W$ as

$$
\widetilde{W}=\left(\begin{array}{cc}
\widetilde{Q}_{1} & \widetilde{Q}_{0} \\
\widetilde{Q}_{0} & \widetilde{Q}_{-1}
\end{array}\right) .
$$

From now on, we give both first and second type Cassini identities for the conjugate Fibonacci and Lucas octonions.

Theorem 2.6. The identities

$$
\begin{aligned}
& \widetilde{Q}_{n-1} \widetilde{Q}_{n+1}-\widetilde{Q}_{n}^{2}=(-1)^{n}\left(2-2 e_{2}-2 e_{3}-4 e_{4}-20 e_{5}-22 e_{6}-23 e_{7}\right) \\
& \widetilde{Q}_{n+1} \widetilde{Q}_{n-1}-\widetilde{Q}_{n}^{2}=(-1)^{n}\left(2-2 e_{1}-4 e_{2}-6 e_{3}-10 e_{4}-2 e_{5}-8 e_{6}-35 e_{7}\right) \\
& \widetilde{H}_{n-1} \widetilde{H}_{n+1}-\widetilde{H}_{n}^{2}=5(-1)^{n-1}\left(2-2 e_{2}-2 e_{3}-4 e_{4}-20 e_{5}-22 e_{6}-23 e_{7}\right)
\end{aligned}
$$

and

$$
\widetilde{H}_{n+1} \widetilde{H}_{n-1}-\widetilde{H}_{n}^{2}=5(-1)^{n-1}\left(2-2 e_{1}-4 e_{2}-6 e_{3}-10 e_{4}-2 e_{5}-8 e_{6}-35 e_{7}\right)
$$

holds for $n \geq 1$.

Proof. The identity

$$
\begin{aligned}
\widetilde{W} U^{n} & =\left(\begin{array}{cc}
\widetilde{Q}_{1} & \widetilde{Q}_{0} \\
\widetilde{Q}_{0} & \widetilde{Q}_{-1}
\end{array}\right)\left(\begin{array}{cc}
F_{n+1} & F_{n} \\
F_{n} & F_{n-1}
\end{array}\right) \\
& =\left(\begin{array}{cc}
\widetilde{Q}_{n+1} & \widetilde{Q}_{n} \\
\widetilde{Q}_{n} & \widetilde{Q}_{n-1}
\end{array}\right) .
\end{aligned}
$$

After taking determinant according to the first and second rules, we obtain first and second identities. To obtain the third and fourth ones, we follow the proofs of Theorem 2.1 and Theorem 2.2.

In order to cut unnecessary repetition, we get the following identities together with the matrices $W, \widetilde{W}$ and $U$.

Theorem 2.7. For the integers $m$ and $n$,

1) The identity $\widetilde{W}\left(U^{m+n}\right) W=\left(\widetilde{W} U^{m}\right)\left(U^{n} W\right)$ yields that

$$
Q_{m+n+1}-\sum_{s=1}^{7} Q_{m+n+1+s} e_{s}=\widetilde{Q}_{m+1} Q_{n+1}+\widetilde{Q}_{m} Q_{n} .
$$

2) The identity $\left(\widetilde{W} U^{m+n}\right) W=\left(\widetilde{W} U^{m}\right)\left(U^{n} W\right)$ gives

$$
\sum_{s=0}^{7} \widetilde{Q}_{m+n+1+s} e_{s}=\widetilde{Q}_{m+1} Q_{n+1}+\widetilde{Q}_{m} Q_{n} .
$$

3) By the identities $\left(W U^{m+n}\right) \widetilde{W}=\left(W U^{m}\right)\left(U^{n} \widetilde{W}\right)$ and $W\left(U^{m+n} \widetilde{W}\right)=\left(W U^{m}\right)\left(U^{n} \widetilde{W}\right)$

$$
\begin{aligned}
\sum_{s=0}^{7} \widetilde{Q}_{m+n+1+s} e_{s} & =Q_{m+1} \widetilde{Q}_{n+1}+Q_{m} \widetilde{Q}_{n} \\
Q_{m+n+1}-\sum_{s=1}^{7} Q_{m+n+1+s} e_{s} & =Q_{m+1} \widetilde{Q}_{n+1}+Q_{m} \widetilde{Q}_{n}
\end{aligned}
$$

hold, respectively. 


\section{Acknowledgement}

The authors are graful for valuable remarks and comments of referees.

\section{References}

[1] Irmak, N.: More identities for Fibonacci and Lucas quaternions. Comm. Faculty of Science University of AnkaraSeries A1 Mathematics and Statistics, 69(1), 369-375 (2020)

[2] Kecilioglu O., Akkus I: The Fibonacci octonions. Adv. Appl. Clifford Algebras. 25, 151-158 (2015).

[3] Koshy T.: Fibonacci and Lucas numbers with applications. Newyork (2001).

[4] Morais J. P., Georgiev S., Spröbig W.: Real Quaternionic Calculus Handbook. Birkhauser. London (2014).

\section{Affiliations}

NURETTIN IRMAK

AdDress: Niğde Ömer Halisdemir University, Art and Science Faculty, Mathematics Department, Niğde, Turkey E-MAIL: irmaknurettin@gmail.com, nirmak@ohu.edu.tr

ORCID ID:0000-0003-0409-4342

ABDULLAH AÇIKEL

AdDRESS: Hatay Mustafa Kemal University, Hassa Vocational School, Hatay, Turkey

E-MAIL: aacikel@mku.edu.tr

ORCID ID:0000-0002-4819-9384 